\title{
Strategies for Palliative Care in Advanced Colorectal Cancer
}

\author{
Matthew R. Dixona Michael J. Stamos ${ }^{b}$ \\ aDepartment of General Surgery, Harbor-UCLA Medical Center, Torrance, Calif., b Division of Colon and \\ Rectal Surgery, Irvine College of Medicine, University of California, Orange, Calif., USA
}

\section{Key Words}

Palliation · Stage 4 cancer - Colorectal cancer · Palliative care

\begin{abstract}
Palliative care has appropriately been receiving increased attention in recent years. From the surgeon's standpoint, therapy is considered palliative when resection of all known tumor sites is no longer possible or advisable. Since a cure, as commonly defined, is not possible, the goal of treatment and eventually the success of therapy becomes judged by the control of symptoms and alleviation of suffering. Providing optimal palliative care for the patient with advanced colorectal cancer is a complex and challenging process. The process of providing palliative care may be a departure from the traditional surgical satisfaction derived from the complete excision of a malignancy, but surgeons achieving excellence in palliative care will likely find this a rewarding endeavor.
\end{abstract}

Copyright $@ 2004$ S. Karger AG, Basel

\section{Palliative Care and Surgery}

Interest in improving the ability to provide palliative care has increased among many different medical specialties. The American College of Surgeons has begun an initiative to incorporate awareness and knowledge of this field among surgical practitioners. The College has recently required palliative care to be included in residency training and developed a task force to facilitate clinical practice initiatives [1]. Palliative care has been defined by the American Academy of Hospice and Palliative Medicine as providing care 'focused on alleviating suffering and promoting quality of life. Major concerns are pain and symptom management, information sharing and advance care planning, psychological and spiritual support, and coordination of care' [2]. When colon and rectal cancer is considered, the surgeon has the ability to become a leader in providing and facilitating the delivery of palliative care. This is particularly important when one considers that as many as $20-25 \%$ of patients with colon and rectal cancer present with evidence of metastatic disease [3]. From a surgical standpoint in colorectal cancer, therapy may be considered palliative when the resection of all known oncologic disease is no longer possible. Surgeons frequently decide which patients are candidates for aggressive surgical resection of metastases. Patients with

Michael J. Stamos, Professor of Surgery, University of California

Irvine College of Medicine, Chief, Division of Colon and Rectal Surgery

University of California-Irvine Medical Center

101 The City Drive, Building 55, Orange, CA 92868 (USA)

Tel. +1 7144568511 , Fax +1 714456 6027, E-Mail mstamos@uci.edu
Fax +4161306 1234 E-Mail karger@karger.ch www.karger.com
(ㄷ) 2004 S. Karger AG, Basel

Accessible online at: www. karger.com/dsu 
unresectable metastatic disease should be treated with a different emphasis than colorectal cancer patients with resectable disease. This requires, first, a paradigm shift in assessing the goals of treatment by the surgeon. Since the cure of disease is not possible, the goal of treatment and essentially the success of therapy becomes judged by control of symptoms and alleviating suffering, which some have suggested are perhaps not adequately emphasized in surgical training [4]. Palliative care may take on a number of forms and may even include aggressive surgical resection for palliation of symptoms. This review will begin with ideas regarding how to develop a treatment plan for patients with metastatic disease, continue with a discussion of nonsurgical palliative interventions and conclude with a description of surgical concepts of palliation.

\section{Developing a Treatment Plan for the Patient with Advanced Disease}

When faced with a patient with colorectal cancer and metastatic disease, the first decision should be whether the patient is a candidate for a potentially curable resection. This would imply resection of the primary along with any known metastases. A complete discussion of the complexities involved in this decision process is not possible in this review, but a clear survival benefit has been shown when isolated hepatic and/or pulmonary metastases are resected [5]. Palliative care is reserved for the remaining patients considered to have unresectable cancer, disseminated metastatic cancer or patients unwilling to undergo extirpative surgery.

Designing a treatment plan for these patients should begin with a frank discussion with the patient and family regarding the situation. An assessment of current functional status and degree of symptoms from the primary tumor should be performed. Patient preferences regarding the degree of intervention desired should be sought. This should also serve as an opportunity to initiate thoughts regarding end-of-life preferences, including code status, if these have not previously been addressed. The authors feel that possessing some idea of the 'survival potential' for a given patient with metastatic disease may prove useful to both the physician and family in choosing the most appropriate treatment course. In published studies involving retrospective evaluation of patients presenting with stage IV colon and rectal cancer, the authors have identified some predictors of survival. Patients with higher levels of CEA and lower levels of albumin were found to have particularly short survival times [6]. Additionally, quantifying the amount of hepatic infiltration by the tumor has been shown to be significantly predictive of survival times [7].

Ideally, goals for treatment, clearly understood by both the surgeon and family, should emerge from these discussions with the family. These goals should be tailored to each patient. Patients with higher functioning status and physical reserves experiencing significant discomfort may benefit from aggressive surgical resection for palliation if desired. Conversely, patients with poorer prognosis may be treated most appropriately by nonsurgical palliation with possible hospice referral. The surgeon plays a unique role in what is ideally a multi-disciplinary effort to provide the best palliative care. The surgeon may counsel the patient on the options available and provide honest expectations of what these options may entail for the patient.

\section{Nonsurgical Methods of Palliation}

The available nonsurgical methods of palliation in colon and rectal cancer are focused on the relief of symptoms as well as the relief of pain. When patients have presented acutely with symptoms such as obstruction, they have historically been treated with urgent operation, usually involving the placement of a stoma. The ability to relieve obstruction without open operation will often allow the conversion of an urgent operation to an elective one with an appropriate preoperative bowel preparation. From a palliative sense, these procedures have also allowed the possibility of a more complete preoperative workup to be performed, often allowing the extent of disease to be identified and, in some cases, open operation to be avoided altogether. This section will examine the nonsurgical methods of palliation currently available.

\section{Endorectal Metallic Stent Placement}

Self-expanding metallic stents were initially introduced for use in the biliary tree and esophagus and have been subsequently adapted for use in relieving large bowel obstruction. The first case reports of endorectal stenting date back to $1991[8,9]$; since that time a number of retrospective studies have been published confirming a generally high success rate of placement with minimal complications [10-15]. Success rates in these reports have varied but have generally been greater than $80 \%$. Complications that have been reported include perforation, migration, tenesmus, rectal bleeding and stent overgrowth. Metallic stents have been successfully placed under both fluoro- 
scopic and endoscopic guidance. The decision regarding which modality to use in placement may be largely based upon institutional preference and familiarity. There is some evidence to suggest that a lesion located more proximally (e.g., transverse colon) may be more successfully reached endoscopically. In order to utilize an endorectal stent, the lesion must be located far enough above the dentate line to obtain a clear margin once the stent is deployed. This usually implies the lesion is further than $6 \mathrm{~cm}$ from the anal verge. A guidewire must be able to be passed proximally through the obstruction in order to guide stent deployment.

Several studies have attempted to compare the usage of endorectal stents with emergency surgery in patients presenting with left-sided colonic obstruction [16-18]. These retrospective reviews have shown a shorter hospital stay, decreased utilization of intensive care and a lower rate of the placement of stomas, when stents were used to acutely relieve obstruction. Additionally, a recent article described outcome data prospectively in 52 patients with colorectal obstruction treated with stents [19]. In this group of patients, stents were successfully inserted in 50 of the 52 patients and the complication rate was $25 \%$. There was one perforation which occurred, but remaining complications were fairly minor with migration being the most common.

\section{Laser Recanalization}

Although many of the most recent studies have focused on the usage of endorectal stents for palliation of obstruction, the first reports of nonoperative management of malignant obstruction utilizing laser photocoagulation actually precede the use of stents and date back to the early 1980s [20, 21]. Modern laser therapy incorporates the use of either endoscopic neodymium:yttrium aluminum garnet (Nd:YAG) or a $\mathrm{CO}_{2}$ laser. Lasers have been shown to be effective in a number of reports in the control of obstructive symptoms and bleeding [22-24]. Palliative laser therapy frequently involves multiple treatments over a period of weeks. It does have the advantage of effectively decreasing the occurrence of bleeding. This modality may be most appropriately utilized in patients without total obstruction who complain of a significant component of bleeding. Patients with complete obstruction may be more effectively treated with an endorectal stent. This is supported by findings of a study that examined a group of 219 patients treated with endoscopic laser therapy for palliation [25]. The authors separated the patients into subgroups in an attempt to identify which patients may be best palliated in this way. Patients with bleeding tended to achieve higher rates of long-term success with laser therapy than those with predominantly obstructive symptoms ( 82 vs. $65 \%$ ). Patients with bleeding as the predominant symptom also required fewer sessions to achieve palliation than those with obstructive symptoms. Additionally, higher rates of complications were seen in those patients presenting with circumferential tumors who were palliated with laser therapy. The authors conclude that endoscopic laser therapy is best suited as a treatment of last resort for those with symptoms of bleeding. There is also some evidence to suggest that laser therapy becomes less effective as patients survive longer, leaving one author to maintain that the best current indications for endoscopic laser therapy are to treat bleeding and tenesmus in rectal cancer patients with the most advanced disease and shortest life expectancy [26]. It may also be used in combination with other palliative measures such as stenting or radiation.

\section{Radiation Therapy}

Radiation therapy has assumed an integral role in the treatment of colon and rectal malignancy when combined with potentially curative resection and may be used in either an adjuvant or neoadjuvant manner. The use of neoadjuvant therapy has been used for low rectal cancer and not uncommonly, patients demonstrate complete responses. One study treated 118 patients with potentially resectable low-rectal cancer with preoperative radiation and chemotherapy [27]. This series identified a significant number of patients (26.2\%) with a complete response who avoided subsequent surgery completely. Given the effectiveness of radiation therapy when used to establish local control of colorectal malignancy, sometimes without operation, it is not surprising that it would play a major role in palliation. Radiation therapy may be useful both for patients presenting initially with advanced disease as well as in those patients with recurrent disease, even for those who may have been treated with prior pelvic radiation. One chief advantage of radiation therapy lies in its ability to affect pain which is likely caused by pelvic nerve invasion by tumor. This symptom is not improved with other palliative treatments such as stent placement or laser therapy.

Using either external beam radiation or intraoperative radiotherapy to palliate patients presenting with incurable colorectal cancer has been described for over 40 years $[28,29]$. Patients may be treated with $4,500 \mathrm{cGy}$ delivered by external beam radiation over a period of several weeks. If the small bowel may be secured or known to remain outside of the dose field, higher levels of radiation may be 
used. Many techniques have been employed in attempts to facilitate this including pelvic displacement prosthesis [30], omental flaps and slings. Additionally, intra-operative radiation therapy may be used alone or in conjunction with external beam radiation [31].

Patients who have previously undergone resection may present with painful recurrences in need of palliation. Although patients previously treated with radiation typically receive what has historically been considered a lifetime dose of pelvic radiation, there is some evidence to suggest that re-irradiation therapy may be an appropriate option to consider in this group of patients. One study retrospectively examined the results of 52 patients receiving re-irradiation for palliation [32]. Patients received between 19.8-40.8 Gy, and the authors reported effective palliation of bleeding and pain. All patients reported initial palliation of bleeding, and palliation of pain was achieved in $65 \%$. Re-irradiation has also been combined with hyperthermia in one retrospective study in order to achieve palliation and $72 \%$ of the patients in this group achieved a good or complete palliative effect [33]. Although hyperthermia may emerge as a useful adjunct to palliative radiation therapy, the authors do conclude that the value of the addition of hyperthermia may not be clearly delineated until more extensive randomized testing is performed.

While radiation therapy does provide effective palliation for symptoms such as pain and bleeding, the decision to begin palliative radiation therapy should be considered in the context of the individual patient with the recognition that the initiation of palliative radiation impacts the ability to maximally utilize palliative chemotherapy [34]. Palliative chemotherapy may need to be reduced by up to $25 \%$ in order to accommodate the additional toxicity involved with combined radiation and chemotherapy. Therefore, this approach does palliate local symptoms at the expense of optimal systemic palliation, and is probably best suited for patients with the most local symptoms.

\section{Chemotherapy}

Success in treating systemic disease in resectable colorectal malignancies with chemotherapy has led to its application in palliation. This has been described for many years, with the first reports including 5-fluorouracil (5-FU) [35]. Treating systemic disease with chemotherapy may double the survival of patients with metastatic disease when compared to those who are not treated [36]. The standard treatment continues to remain 5-FU based, although there are several emerging chemotherapeutic modalities available for palliation [37]. Irinotecan-CPT11 is a topoisomerase inhibitor and has been found to produce a response rate between 10.4 and $27 \%$ when used with patients with relapsed and refractory colorectal cancer. Potential side effects include neutropenia, diarrhea and vomiting. Lack of a prior response to 5-FU does not seem to predict failure of irinotecan-CPT11. Another newer agent that has proven useful in the palliation of colorectal cancer is oxaliplatin. This is a third generation platinum complex with the most common side effects being nausea, vomiting, peripheral neuropathy and sporadic hematologic toxicity. Several trials have shown this to provide a significant improvement in survival of patients with metastatic disease when these new medications are added to a 5-FU regimen [38].

Orally administered 5-FU prodrugs are now available for use and may be similar to 5-FU in efficacy. These include tegafur/uracil and capecitabine [39]. Advantages for palliation include ease of administration and lack of complications related to intravenous access. Additionally, some phase III trials have suggested that capecitabine may have a lower incidence of side effects such as nausea, diarrhea, alopecia and neutropenia when compared with traditional bolus 5-FU therapy. These differences may potentially increase the quality of life for patients receiving palliative chemotherapy.

One combination involving oxaliplatin with infused 5FU and leucovorin (FOLFOX) has been compared with regimens utilizing 5-FU with irinotecan, and irinotecan and oxaliplatin [40]. In this randomized controlled trial involving 795 patients, a significantly higher response rate and overall survival was demonstrated in patients randomized to FOLFOX treatment, leading the authors to suggest that this should become first-line therapy for patients with stage IV colorectal cancer. The patients randomized to FOLFOX displayed a median survival of 19.5 months which is the longest survival reported in a phase III trial in this group of patients. The active ongoing research in identifying optimal chemotherapeutics for metastatic colorectal cancer and the significant survival benefit achieved with chemotherapy underscores the value of a multi-disciplinary approach to the management of these patients. Input from medical oncologists should be considered when planning the treatment sequence and coordinating interventions for palliation.

\section{Pain Management}

Identifying and treating the pain associated with advanced colorectal cancer is an imperative part of management. The proper and adequate control of pain is an out- 
come that is increasingly expected by both patients and families as well as hospital review committees such as the Joint Commission on Accreditation of Healthcare Organizations [41]. Pain control is particularly important for patients with metastatic cancer who may commonly suffer from pelvic pain related to the primary tumor or from symptoms of metastases.

The first-line treatment for pain are usually oral opioids which are effective for many patients. These should be prescribed adequately for this group of patients. Fears regarding the potential for addiction and liability related to narcotic prescription are generally unfounded in the context of palliative care. Some authors have suggested that the inadequate treatment of pain is actually an area of concern for potential physician liability in palliative care [42]. Many states, such as California, have provisions such as the 11159.2 exemption that allow any physician to prescribe narcotics in the context of palliative care without special requirements which are in place for other patients.

When pain is not relieved with opioids, one may consider trying oral medications such as amitriptyline or gabapentin for neuropathic pain. A multidisciplinary approach utilizing pain care specialists, usually from the department of anesthesiology, may prove useful for patients with pain that is most difficult to control. Epidural catheter placement may allow more effective pain control in select patients. Neuroablative techniques, such as open or percutaneous cordotomy, block pain transmission through the spinothalamic tract of the spinal cord and may be considered for patients with the most refractory pain [43].

\section{Surgical Palliation}

Surgical palliation remains an active area of debate and controversy in the surgical literature. Many advances have been made that allow more to be accomplished surgically, often with less debilitation that is often incurred with open laparotomy. This section will address the issues regarding the management of the primary tumor, the surgical options available for resection including the growth of limited or transanal resection, and conclude with an overview of the currently available minimally invasive palliative procedures.

\section{Management of the Primary Tumor}

There is currently no consensus in the literature concerning the management of the primary tumor when treating patients with incurable colon and rectal cancer. Traditionally, resection has often been advocated to eliminate the source of future symptoms such as bleeding and obstruction. The mortality in the setting of emergency surgery for stage IV disease has also been quoted to be as high as $27 \%$, lending additional support for elective removal of the primary tumor [44]. With the advent of the previously discussed nonsurgical interventions, many symptoms can be safely palliated nonoperatively. Additionally, in the past, patients presenting with malignant large bowel obstruction were often taken emergently to the operating room, facilitating initial surgical management. Now that patients may be effectively temporized with a stent, surgeons are faced with deciding whether to operate on the primary tumor in this group of patients.

Central to the difficulty in this discussion is the realization that patients presenting for palliative care often display a heterogeneity in survival time as well as overall physical condition and fitness for major surgery making some type of stratification necessary to generate appropriate recommendations. Several recent reviews have attempted to determine which patients should undergo elective resection with incurable stage IV disease. One recent nonrandomized review identified 127 patients with stage IV disease who underwent elective resection of a primary tumor (with additional known metastases which were not resected) and compared them with 103 patients with metastatic disease over the same time period treated nonoperatively [45]. This study found a significantly longer survival in those undergoing resection. Among this group, patients with only one site of distant metastases, metastases confined to the liver and less than 25\% hepatic replacement by tumor showed significantly longer survival times. Support for nonoperative management has also been documented for patients with unresectable disease and minimally symptomatic colorectal cancer [46]. The authors of this prospective review followed 24 patients with unresectable disease and deferred initial management of the primary tumor, beginning with systemic chemotherapy. Four patients in this group did develop bowel obstructions which were managed surgically in two and with stent placement in another two. The remaining patients did not require surgery for any acute complications, leading the authors to advocate that patients with metastatic disease are more likely to die of systemic disease than from the primary tumor. Another review of palliative surgery performed for patients with peritoneal dissemination from colorectal cancer presenting with obstruction suggested the amount of ascites may be one prognostic factor to consider [47]. Patients in this group 
with more than $100 \mathrm{ml}$ of ascites displayed a markedly poor success rate when surgery was attempted for palliation of obstruction.

Given the lack of randomized prospective data in this area, the surgeon providing palliative care for the patient with metastatic colorectal cancer should consider the age and performance status of the patient, current symptoms, survival potential based on factors including CEA, albumin and quantity of metastases and patient preferences. Palliative care may not necessarily entail resection of the primary tumor and this is a choice that should be made in the context of the individual patient. The elimination of a pelvic source of tumor is certainly an advantage for patients with limited metastatic disease because it possibly spares future pelvic pain, ureteral obstruction and the potential decrease in quality of life. The basis of this decision may partially involve a clinical judgment regarding which focus of disease is most life-threatening. Younger patients with less widespread disease and very symptomatic tumors may be offered aggressive resection as a palliative option while the majority of older patients with more advanced disease may avoid routine laparotomy and be more effectively treated with immediate chemotherapy.

\section{Palliative Open Resection}

Once the decision has been made to perform resection for palliation, the surgeon has a number of different options. The most commonly performed open operations for palliation include abdominoperineal resection (APR), pelvic exenteration, Hartman's procedure and low anterior resection (LAR).

The most radical surgical option used in the palliation of colorectal cancer is pelvic exenteration. This may be utilized when a primary tumor, most commonly rectal, has invaded structures in the pelvis such as the bladder, vagina or pelvis. These operations may be further classified as total or posterior, depending on whether the rectum, bladder and internal reproductive organs are removed or only the rectum and internal reproductive organs are removed. Additionally, an extended pelvic exenteration involves resection of the bony pelvis. Although a number of reviews have documented the use of pelvic exenteration as a palliative option [48-50], it should likely be reserved for a small group of the most fit patients as the literature also uniformly agrees on the significant morbidity associated with its use. This operation places a tremendous physiologic challenge upon the patient with reviews estimating blood losses between 3,800 and $5,000 \mathrm{~cm}^{3}$ [49] and operating times between $3.5-10 \mathrm{~h}$. Postoperatively, patients may expect significant morbidi- ty $(45-60 \%)$ with the probable likelihood of readmissions. One author has advocated the consideration of the cost of these readmissions in terms of percent remaining days of life spent in the hospital [50]. This operation may be most effective for patients with severe central, not radicular, pelvic pain and disabling perineal wounds with such significant nursing care requirements that quality of life is severely disadvantaged. Given the morbidity and postoperative recovery entailed, from a palliative point of view, this operation is most suited for those patients with a higher potential for survival.

Patients with less advanced primary colon or rectal tumors may be treated with APR, Hartman's procedure, LAR or colectomy. Operative approach depends largely on the location and size of the primary tumor. Some authors have advocated the use of Hartman's procedure over an APR for palliation because it avoids the creation of a perineal wound and the morbidity and pain that this frequently causes [51]. APR may be the only palliative option for patients with the lowest tumors including those involving the sphincters. One review examining 80 patients treated at Memorial Sloan-Kettering with radical surgery followed by chemotherapy in stage IV rectal cancer patients used LAR in 65 patients, APR in 11 patients and Hartman's resection in another 4 patients [52].

\section{Local Excision}

Another important treatment option that may be considered when providing palliative care for patients with advanced rectal cancer is local palliative excision. This may be performed with a urologic resectoscope or tumor may be removed using cryosurgery $[53,54]$.

This minimally invasive modality may be used on patients with significant comorbidities that preclude the option of open surgery. In contrast to stent placement and laser recanalization which provide palliation of symptoms but generally leave the tumor relatively intact, these methods allow local control of the tumor to be achieved. Transanal resection using a resectoscope allows tumors to be removed under direct vision with electrocautery while continuous irrigation facilitates visualization. A retrospective review compared the palliation achieved with endoscopic transanal resection (ETAR) with transabdominal resection [55]. This group of 24 patients who underwent ETAR were matched with 25 patients who underwent palliative LAR, APR or Hartman's procedure. Survival was similar in the two groups and there was a much higher morbidity in the group receiving open surgery as well as significantly higher stoma rate. The authors suggest that ETAR may be considered as a palliative option 
for low fixed rectal tumors that may be difficult to treat with LAR and for very elderly patients who may not be candidates for general anesthesia.

\section{Laparoscopic Palliation}

The development of laparoscopy has also increased surgical options for palliation in advanced colorectal cancer. While concerns of port-site recurrence have initially limited the use of laparoscopy to clinical trials for attempted curative resection, this is not an issue when applied to surgeries with palliative intent. Laparoscopy may be used to effectively provide diversion with either colostomy or ileostomy placement. One technique employs a trocar inserted at the umbilicus with a second trocar inserted at the stoma site [56]. The bowel may be mobilized before the stoma site is created with the trocar by pulling and exteriorizing the bowel at the site of insertion. These laparoscopic techniques allow the same effective diversion associated with open surgery to be created with a significantly less invasive procedure.

Laparoscopic palliation for colorectal cancer was reviewed in a study involving thirty patients [57]. The authors utilized the laparoscopic approach to perform both uncomplicated colon resections, resections of two colonic areas (patients with double lesion) as well as laparoscopic colostomy placement. Of the 30 patients, there were only 3 patients who required an intra-operative con- version to an open procedure. Patients in this series receiving laparoscopic stoma placement had a median operating time of $60 \mathrm{~min}$ with a median blood loss of only $50 \mathrm{ml}$.

\section{Conclusion}

Providing optimal palliative care for the patient with advanced colorectal cancer is a complex and challenging process. The surgeon may play a unique role in weighing the palliative modalities available and providing honest and accurate information to the patient regarding what he/she may expect. The best palliative care will likely come from a multidisciplinary team that individualizes the treatment plan in accordance with the patient's wishes, allowing symptoms to be maximally treated, lifespan to be optimized and hospital stay (particularly the ratio number of inpatient days to number of days of remaining survival) to be minimized. The goals involved with providing palliative surgical care may be a departure from the traditional surgical satisfaction derived from the complete excision of malignancy, but surgeons achieving excellence in palliation will likely find this a rewarding endeavor, given the potential positive impact this can have on their patients suffering from advanced colorectal cancer.

\section{References}

1 Weissman DE: Surgery and palliative care. J Palliat Med 2003;4:525-526.

2 Gold MF: Comfort, compassion, dignity mark end of life care. Provider 2003;29:20-33.

$\checkmark 3$ Greenlee RT, Murray T, Bolden S, Wingo PA: Cancer Statistics, 2000. CA Cancer J Clin 2000;50:7-33

4 Page DW: Blood, sweat and tears: Are surgeons prepared for the challenges of self-reflection? J Palliat Med 2003;4:625-627.

$\checkmark 5$ Scheele J, Stang R, Altendorfer-Hofman A Paul A: Resection of colorectal liver metastases. World J Surg 1995;19:59-71.

6 Dixon MR, Haukoos JS, Udani SM, et al: CEA and albumin predict survival in patients presenting with advanced colon and rectal cancer. Arch Surg, 2003;138:962-966.

7 Dixon MR, Haukoos JS, Udani SM, et al: Prediction of survival in stage IV colorectal cancer by a computed tomographic scoring system for hepatic metastasis (abstract). J Surg Res 2003; 114:301.

8 Dohmoto M: New method: Endoscopic implantation of rectal stent in palliative treatment of malignant stenosis. Endoscopia Digestiva 1991;3:1507-1512.
Spinelli P, Dal Fante M, Mancini A: Selfexpanding mesh stent for endoscopic palliation of rectal obstructing tumors: A preliminary report. Surg Endosc 1992;6:72-74.

10 Turegano-Fuentes F, Echenagusia-Belda A, Simo-Muerza G, et al: Transanal self-expanding metal stents as an alternative to palliative colostomy in selected patients with malignant obstruction of the left colon. Br J Surg 1998;85: 232-235.

11 Aviv RI, Shyamalan G, Watkinson A, et al: Radiological palliation of malignant colonic obstruction. Clin Radiol 2002;56:347-351.

12 Tejero E, Fernando-Lobato R, Mainar A, et al: Initial results of a new procedure for treatment of malignant obstruction of the left colon. Dis Colon Rectum 1997;40:432-436.

13 Spinelli P, Mancini A: Use of self-expanding metal stents for palliation of rectosigmoid cancer. Gastrointest Endosc 2001;53:203-206.

14 Gukovsky-Reicher S, Lin RM, Sial S, et al: Selfexpandable metal stents in palliation of malignant gastrointestinal obstruction: Review of the current literature data and 5-year experience at Harbor-UCLA Medical Center. MedGenMed 2003;10:16.
15 Arnell T, Stamos MJ, Takahashi P: Colonic stents in colorectal obstruction. Am Surg 1998; 64:986-988.

16 Law WL, Choi HK, Chu KW: Comparison of stenting with emergency surgery as palliative treatment for obstructing primary left-sided colon cancer. Br J Surg 2003;90:1429-1433.

17 Martinez-Santos C, Lobato RF, Fradejas JM: Self-expandable stent before elective surgery vs. emergency surgery for the treatment of malignant colorectal obstructions: Comparison of primary anastamosis and morbidity rates. Dis Colon Rectum 2002;45:401-406.

18 Law WL, Choi HK, Chu KW: Comparison of emergency surgery as palliative treatment for obstructing primary left-sided colorectal cancer. Br J Surg 2003;90:1429-1433.

19 Law WL, Choi HK, Lee YM, et al: Palliation of advanced colorectal obstruction by self-expanding metallic stents: Prospective evaluation of outcomes. Dis Colon Rectum 2004;47:39_ 43

20 Lambert R, Sabhen G: Laser therapy for colonic neoplasms (abstract). Lasers Surg Med 1983;3:147. 
21 Thompson P, Laurence B: Laser photocoagulation in obstructing malignant tumours of the respiratory and gastrointestinal tracts. Med J Aust 1985;142:545-546.

22 Walfisch S, Stern H, Ball S: Use of Nd-YAG laser ablation in colorectal obstruction and palliation in high-risk patients. Dis Colon Rectum 1989;32:1060-1064

-23 Loizoi, Grigg D, Boulos, et al: Endoscopic $\mathrm{Nd}$ :Yag laser treatment of rectosigmoid cancer. Gut 1990;31:812-816.

24 Daneker GW, Carlson GW, Hohn DC, et al: Endoscopic laser recanalization is effective for prevention and treatment of obstruction in sigmoid and rectal cancer. Arch Surg 1991;126. 1348-1351.

-25 Gevers AM, Macken E, Hiele M, Rutgeerts P. Endoscopic laser therapy for palliation of patients with distal colorectal carcinoma: Analysis of factors influencing long-term outcome. Gastrointest Endosc 2000;51:580-585.

26 Kimmey MB: Endoscopic methods (other than stents) for palliation of rectal carcinoma. J Gastrointest Surg 2004;8:270-273.

-27 Habr-Gama A, de Souza PMSB, Ribeiro U, Nadalin W, et al: Low rectal cancer; impact of radiation and chemotherapy on surgical treatment. Dis Colon Rectum 1998;41:1087-1095.

28 Wang CC, Schulz MO: The role of radiation therapy in the management of carcinoma of the sigmoid, rectosigmoid, and rectum. Radiology 1962;79:1.

-29 Gunderson LL, Cohen AM, Welch CE: Residual, inoperable or recurrent colorectal cancer. Interaction of surgery and radiotherapy. Am J Surg 1980;139:518-525.

>30 Edington HD, Hancock S, Coe FL, Sugarbaker PH: Preliminary report of a new treatment strategy for advanced pelvic malignancy: Surgical resection and radiation therapy using afterloading catheters plus an inflatable displacement prosthesis in the treatment of advanced primary and recurrent rectal cancer. Surgery 1986;3:494-499.

-31 Minsky BD, Cohen AM, Faas D, et al: Intraoperative brachytherapy alone for incomplete resected recurrent rectal cancer. Radiat Oncol 1991;21:115-120.
32 Lingareddy V, Ahmad NR, Mohuiddin M: Palliative reirradition for recurrent rectal cancer. Int J Radiat Oncol 1997;38:785-790.

-33 Jufferman JM, Hanssens PE, Putten WL, et al: Reirradiation and hyperthermia in rectal carcinoma: A retrospective study on palliative effect. Cancer 2003;98:1759-1766.

34 Saltz LB: Palliative management of rectal cancer: The roles of chemotherapy and radiation therapy. J Gastrointest Surg 2004;8:274-276.

35 Erlichman C, Fine S, Wong A, Elhakim T: A randomized trial of fluorouracil and folinic acid in patients with metastatic colorectal carcinoma. J Clin Oncol 1988;6:469-475.

-36 Scheithauer W, Rosen H, Kornek GV, et al: Randomised comparison of combination chemotherapy plus supportive care with supportive care alone in patients with metastatic colorectal cancer. Br J Med 1993;306:752-755.

37 Beretta GD, Pessi MA, Poletti P, Mosconi S, Labianca R: New drugs and combinations in the palliative treatment of colon and rectal cancer. Eur J Surg Oncol 2001;27:595-600.

-38 Folprecht G, Kohne $\mathrm{CH}$ : The role of new agents in the treatment of colorectal cancer. Oncology 2004;66:1-17.

39 Braun AH, Acterrath W, Wilke H, et al: New systemic frontline treatment for metastastic colorectal carcinoma. Cancer 2004;100:15581577.

40 Goldberg RM, et al: A Randomized controlled trial of fluorouracil plus leucovorin, irinotecan, and oxaliplatin combinations in patients with previously untreated metastatic colorectal cancer. J Clin Oncol 2004;22:23-30.

41 Pain assessment and management standards hospitals. JCAHO requirement. http://www. jcrinc.com/subscribers/perspectives.asp?durki $=3243$.

42 Baluss ME, Lee KF: Legal considerations for palliative care in surgical practice. J Am Coll Surg 2003;197:323-330.

43 Mullan S, Hekmatpanah J, Dobben G, et al: Percutaneous intramedullary cordotomy utilizing the unique unipolar anodal electrolytic system. J Neurosurg 1992;22:548.

44 Stamatakis J, Thompson M, Chave H, Kinsman R: National audit of bowel obstruction due to colorectal cancer April 1998-March 1999. London, The Association of Coloproctology of Great Britain and Ireland 2000.
5 Ruo L, Gougoutas C, Paty P, et al: Elective bowel resection for incurable stage IV colorectal cancer: Prognostic variables for asymptomatic patients. J Am Coll Surg 2003;5:722-728.

46 Sarela AI, Guthrie JA, Seymour MT, et al: Non-operative management of the primary tumor in patients with incurable stage IV colorectal cancer. Br J Surg 2001;88:1352-1356.

47 Higashi $\mathrm{H}$, et al: Factors affecting successful palliative surgery for malignant bowel obstruction due to peritoneal dissemination from colorectal cancer. Jpn J Clin Oncol 2003;33:357359.

48 Finlayson CA, Eisenberg BL: Palliative pelvic exenteration: Patient selection and results. Oncology 1996;10:479-483.

49 Moffat FL, Falk RE: Radical Surgery for extensive rectal cancer: Is it worthwhile? Recent Results Cancer Res 1998;146:73-83.

50 Kakuda JT, Lamont JP, Chu DZ, Paz IB: The role of pelvic exenteration in the management of recurrent rectal cancer. Am J Surg 2003;186: 660-664.

51 Heah SM, Eu KW, Ho YH, Leong AFPK, Seow-Choen F: Hartman's procedure vs. abdominoperineal resection for palliation of advanced low rectal cancer. Dis Colon Rectum 1997;11:1313-1317.

52 Nash GM, Saltz LB, Kemeny NE, et al: Radical resection of rectal cancer primary tumor provides effective local therapy in patients with stage IV disease. Ann Surg Oncol 2002;9:954960.

53 Hamy A, Tuech JJ, Pessaux, et al: Palliation of carcinoma of the rectum using the urologic resectoscope. Surg Endosc 2003; 17:627-631.

54 Meijer S, Rahusen FD, van der Plas LG: Palliative cryosurgery for rectal carcinoma. Int $\mathrm{J}$ Colorectal Dis 1999;14:177-180.

55 Chen H, George BD, Kaufman HS, et al: Endoscopic transanal resection provides palliation equivalent to transabdominal resection in patients with metastatic rectal cancer. J Gastrointest Surg 2001;5:282-286.

56 Lefor AT: The role of laparoscopy in the treatment of intra-abdominal malignancies. Cancer J 2000;6:S159-S168.

57 Milsom JW, Kim SH, Hammerhofer KA, Fazio VW: Laparoscopic colorectal surgery for palliation. Dis Colon Rectum 2000;43:15121516 